\title{
Predictors of caregiving satisfaction in informal caregivers of people with dementia
}

\author{
Carmen de Labra, José C. Millán-Calenti, Ana Buján, Laura Núñez-Naveira, Anders M. \\ Jensen, Merete Charlotte Peersen, Ewa Mojs, Wlodzimierz Samborski, Ana Maseda
}

\begin{abstract}
Objective. The prevalence of dementia is increasing and consequently the demands from families, institutions and healthcare system. Although a substantial amount of research on caregiving has emphasized the negative aspects of caregiving, specifically on caregiver burden and depression, less attention has been paid to the positive aspects of caregiving. The aim of the present work was to study the phenomenon of caregiving satisfaction in informal caregivers of people with dementia by assessing their likely predictors.

Methods. A stress process model was used to study caregiver's satisfaction (measured using the Revised Caregiving Satisfaction Scale) on 101 informal caregivers of patients with dementia in relation to the caregiver's background and context, stress-related factors, and mediators.

Results. The regression model has an adjusted $R^{2}$ of 0.20 , which indicates that having a consanguinity relationship with the care recipient, suffering from lower levels of subjective burden, and managing individuals with severe cognitive impairment are the most important predictors of higher caregiving satisfaction.

Conclusion. Interventions focused on the enhancement of the caregiving satisfaction by increasing the understanding of the disease, should be especially addressed to caregivers without a consanguinity relationship and with high levels of subjective burden, and to those managing care recipients with mild or moderate stages of dementia.
\end{abstract}

Keywords

Positive aspects; Predictors; Burden

\section{Introduction}

That the world's population is aging is a well-recognized fact. According to current estimations, 35.6 million of people worldwide live with dementia, and this number will likely double by 2030 (World Health Organization and Alzheimer's Disease International, 2012).

People with dementia generally require a high level of care, and informal caregivers, primarily spouses and daughters, are the ones who provide this type of care (Brodaty \& Donkin, 2009), playing a very important role in this process. Providing daily care to someone with dementia is not only time consuming, but also a stressful and demanding task. Literature has found many characteristics related with negative aspects of caregiving. There is consistent documentation that caregivers become ill more often compared with those who do not perform this type of service (Kiecolt-Glaser et al., 1991 and Vitaliano et al., 2003) and that caregivers have more physiological and/or psychological problems. Among physiological changes, increased stress hormones and inflammatory markers (Vitaliano et al., 2011 and von Känel et al., 2012), hypertension (Roepke et al., 2011), and metabolic syndrome (Mausbach et al., 2010) were found. As regards psychological problems, depression (Alspaugh et al., 1999, Kiecolt-Glaser et al., 1991 and Schulz et al., 2005) and anxiety (Anthony-Bergstone et al., 1988, Cooper et al., 2007 and Schulz et al., 2005) have been recurrently reported in caregiver's research.

Although these negative outcomes constitute an important part of caregiving research, an area of investigation on caregiving showing the positive potential consequences of the caregiving experience has not been as well researched as negative aspects (Andrén \& Elmståhl, 2005). Many studies addressing these positive experiences have shown how a large number of caregivers could describe one or more positive aspects of caregiving (PAC) when asked (Cohen, Colantonio, \& Vernich, 2002). PAC are typically defined as the rewards and satisfaction derived from the caregiver relationship (Hilgeman, Allen, DeCoster, \& Burgio, 2007). Different aspects of positive consequences have been previously identified: caregiver sense of mastery (Cohen et al., 1994, Lawton et al., 1989 and Pearlin et al., 1990); caregiver enjoyment derived from the present relationship (Cohen et al., 1994 and Pruchno et al., 1990); 
caregiving gratification in observing desirable outcomes, such as the care receiver remaining at home rather than in a specialized facility or appearing to be happy (Motenko, 1989); and satisfaction, sense of meaningfulness and fulfillment from caregiving (Cohen et al., 1994, Lawton et al., 1989, López et al., 2005, Noonan and Tennstedt, 1997 and Tarlow et al., 2004). An overview of studies on PAC indicates consistent positive associations between the positive feeling and variables such as the quality of the relationship before the illness (López et al., 2005), the fact that the caregiver is satisfied with the social support he/she receives (Schulz, Newsom, Fleissner, de Camp, \& Nieboer, 1997), and a negative association with the caregiver's perception of burden (Hilgeman et al., 2007).

The positive aspects of caregiving have been conceptualized differently across studies. It is difficult to define the positive aspects of caregiving since there is no clear definition of this construct (Carbonneau et al., 2010 and Kramer, 1997). Among all of the PAC, the phenomenon of satisfaction derived from the caregiving relationship seems to achieve a high level of importance (Kramer, 1997). Lawton et al. (1989) were the first to introduce the term "satisfaction" as one of the major dimensions of caregiving general positive appraisal, representing subjectively perceived gains from desirable aspects of caregiving. They developed a scale to measure this general appraisal, the Caregiving Satisfaction Scale (CSS) that comprises several aspects that have been included in the literature about PAC: feeling closer to the patient, enjoying being with the patient, boosting the caregiver's self-esteem, delighting in the patient's pleasure, and giving meaning to the caregiver's life.

However, although satisfaction with caregiving has been reported in the literature (Lawton et al., 1989, Lawton et al., 1991, López et al., 2005 and Lundh, 1999), little is known regarding its actual predictors. Using the CSS, López et al. (2005) conducted a study with the aim of determining several factors that could be considered predictors of caregiving satisfaction. They found that higher satisfaction was associated with better previous relationships between the caregivers and the elderly with dependence, with being caregivers voluntarily, with maintaining leisure time, with less need for venting emotions, and with caregivers not working outside the home.

Consistent with this investigation and with the aim of extending our knowledge concerning PAC, the conceptual framework guiding this study is adapted from the stress process model (SPM) of Pearlin et al. (1990) to examine the predictive factors associated with caregiving satisfaction. According to this conceptual model, the caregiver's stress is considered a process that is composed of many conditions, and the outcomes are the result of the interaction of these different conditions. The conceptual framework for this study incorporates variables representing each of the four domains of the model of Pearlin et al. (1990), including the background and context within which the stress occurs, the actual stressors, the mediators, and the caregiver's outcomes or manifestations of stress (Fig. 1). The likely outcomes of the SPM of Pearlin et al. (1990) involve the well-being of the caregiver and their physical and mental health. The majority of research regarding caregivers of patients with dementia has employed the model linked to negative aspects of caregiving ( Crespo \& Fernández-Lansac, 2013). Therefore, our model incorporates a modification to the model of Pearlin et al. with the treatment of what they considered to be personal gain as a positive outcome represented by caregiving satisfaction (see Fig. 1). This variation is consistent with the two-factor model of Lawton et al. (1991), which postulates that the caregiving appraisal could be considered an outcome of caregiving. 


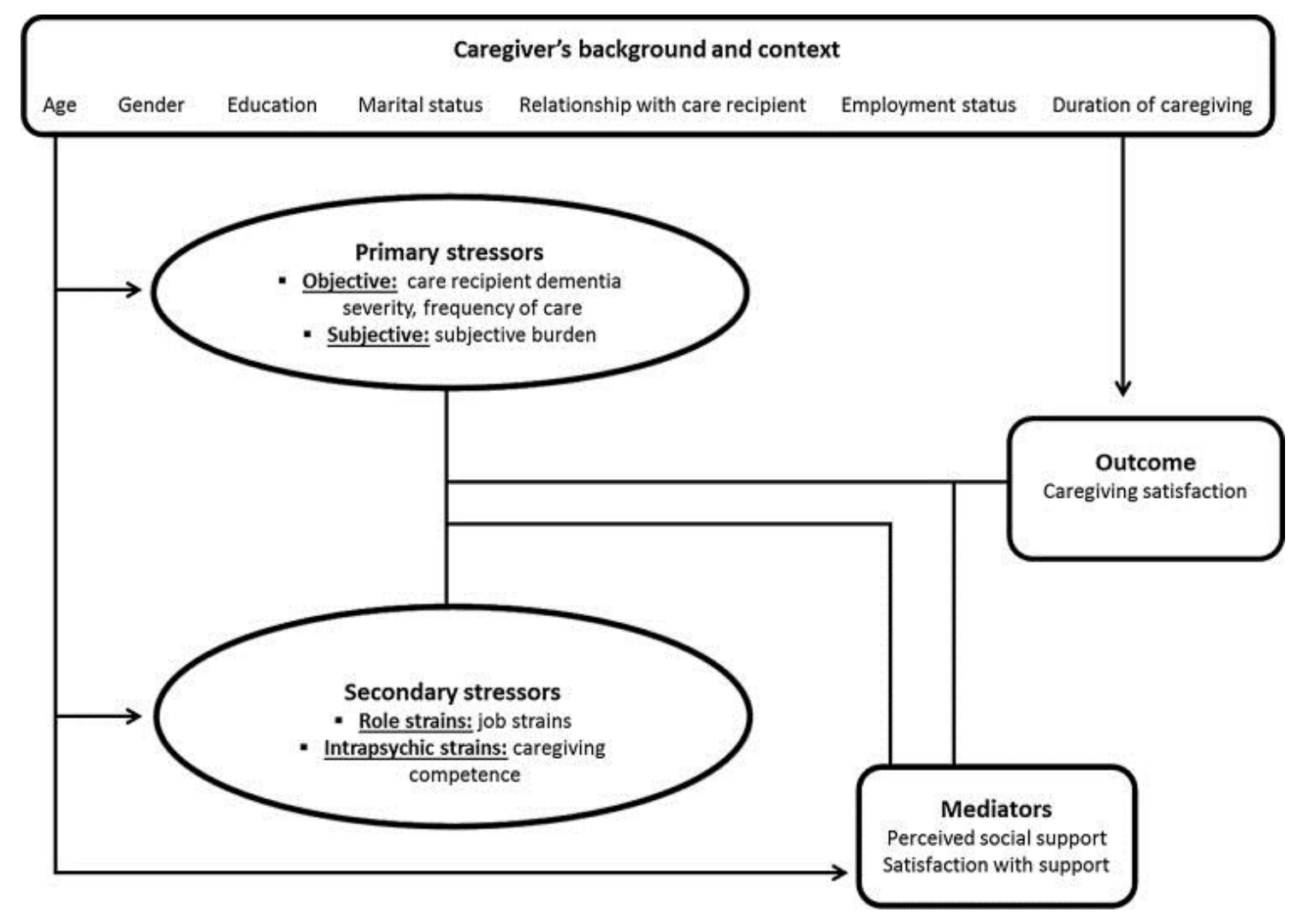

Fig. 1. Conceptual framework used in the current study based on the stress-process model of Pearlin et al. (1990).

The perception of family caregiving as satisfying may serve as a protective factor from the negative consequences associated with providing care (Robertson, Zarit, Duncan, Rovine, \& Femia, 2007). Several studies have shown important relations between positive aspects of caregiving and less likelihood to report depression, burden or poor health (Cohen et al., 2002), positive affect (Lawton et al., 1991), improvement of caregiver well-being (Motenko, 1989 and Noonan and Tennstedt, 1997), less possibilities to actually institutionalize their family members (Mausbach et al., 2004) or to feel the desire to institutionalize them (Cohen et al., 1994). Therefore, knowing the factors that predict caregiver's satisfaction will facilitate the design of interventions focused on these predictors to increase this positive experience, and thus buffering the negative consequences of caregiving.

In summary, the current study uses the caregiver's satisfaction as a potentially important output that is often missed in the literature concerning the SPM. Different aspects of caregiver's background and context characteristics, stress-related factors, and perceived social support were analyzed to examine which variables in each domain can act as predictors of the feeling of satisfaction in caregivers.

\section{Materials and methods}

\subsection{Selection and description of participants}

The sample included 101 informal caregivers of patients with dementia living in the community. The caregivers were recruited from local Alzheimer's associations or adult day care programs from 3 countries: Spain, Poland and Denmark. The inclusion criteria for the study were as follows: (a) taking care of a person diagnosed with dementia by a specialist or a neurologist, according to the criteria of the Classification of Mental and Behavioural Disorders, 10th revision (ICD-10, World Health Organization, 1992), or the Diagnostic and Statistical Manual of Mental Disorders, 4th edition, text revision (DSM-IVTR, American Psychiatric Association, 2000), or the National Institute of Neurological Disorders and Stroke-Alzheimer Disease and Related Disorders Association (NINDS-ADRDA, McKhann et al., 1984); (b) being the primary caregiver in the following aspects: executing basic care tasks for a minimum of 6 weeks, receiving no remuneration for caregiving service, and devoting much time to patient care (Wilson, 1989); (c) suffering a burden according to the 22-item version of the Zarit Burden Interview (ZBI, Zarit, 
Orr, \& Zarit, 1985), using a cut-off point of 24, which was determined to identify family caregivers who are at risk for depression (Schreiner, Morimoto, Arai, \& Zarit, 2006); and (d) agreeing to participate in the study and signing the informed consent. Caregivers were excluded if they did not possess the necessary skills to be assessed (cognitive impairment, illiteracy or severely impaired vision and hearing).

\subsection{Procedure}

The study protocol was approved by the local ethics committees in all three countries according to the specific national guidelines and conformed to the principles of the Declaration of Helsinki. Before data collection, all of the participants were provided information concerning the study and signed the informed consent.

\subsection{Variables and instruments}

\subsubsection{Caregiver's background and context}

The caregivers who were eligible for enrollment in the study were evaluated using a comprehensive assessment by clinical professionals who had extensive experience working with patients diagnosed with dementia and their informal caregivers. A self-elaborated questionnaire collected the following sociodemographic variables: caregiver age, caregiver gender, level of education (low: from 0 to 8 years of education; medium: from 9 to 12 years of education; high: college or higher degree), marital status (with partner: married or cohabiting; without partner: single, divorced/separated, widowed), type of relationship with the care recipient (consanguinity relationship: adult children, sibling, grandchild, nephew/niece; no consanguinity relationship: spouse, children-in-law), employment status (employed; unemployed), and duration of caregiving (total months as caregiver).

\subsubsection{Stress-related factors}

Regarding stress-related factors, the model developed by Pearlin et al. (1990) includes as primary stressors the cognitive, functional and behavioral status of the patients with dementia. To identify and establish the severity of dementia in each patient, the Global Deterioration Scale (GDS) ( Reisberg, Ferris, De Leon, \& Crook, 1982) was used. GDS is a global rating scale that breaks down cognitive function into seven stages, from no cognitive decline to very severe cognitive decline, also including aspects related to functional and behavioral domains. The levels 4 and 5 indicate mild and moderate dementia, whereas levels 6 and 7 indicate moderately severe and severe dementia. Additionally, the frequency of care was evaluated as a primary stressor, considering the number of hours devoted to care per week ( $<20 \mathrm{~h}$ per week; $\geq 20 \mathrm{~h}$ per week). Another indicator of primary stressor, the overload or subjective burden felt by the informal caregivers, was measured using the 22 -item version of the Zarit Burden Interview (ZBI) ( Zarit et al., 1985). The maximum score in this scale is 88 points, with higher scores indicating an increased level of caregiver burden.

With regard to secondary stressors, the likelihood of changing the work shift (assessed through the YES/NO question "Have you changed your working shift in order to take care of your relative?"), and leaving work to take care of a relative (assessed through the YES/NO question "Have you lost or left your work in order to take care of your relative?") were evaluated as role strain, and the sense of competence experienced by the caregiver was measured as intrapsychic strain using the Caregiver Competence Scale (CCS) (Pearlin et al., 1990). With 4 items, this scale measures the caregivers' perceived competence of their own performance in the caregiving tasks, with a maximum score of 16 points and higher scores indicating more feelings of competence.

\subsubsection{Mediators}

Because the role of coping has been extensively evaluated in studies of caregivers (Crespo and Fernández-Lansac, 2013, Haley et al., 1987, López et al., 2005 and Noonan and Tennstedt, 1997), we focused this investigation on the other mediator considered in the caregiver stress model (Pearlin et al., 1990), that is, the social support of caregivers. In particular, we asked through a series of YES/NO questions about the different types of support the caregivers used, such as the help provided by the general practitioner, dementia supervisor, relatives and friends, home services, respite care services, and self-help groups. Additionally, perceived social support and satisfaction with that social support were measured using the Social Support Questionnaire: Short form Revised (SSQRS) (Sarason, Sarason, Shearin, \& Pierce, 1987). In this 6-item questionnaire, each item is a question that requests a two-part answer that assesses the size of the network (maximum score of 9 points with higher scores indicating 
higher network) and the individual's degree of satisfaction with different aspects of support (maximum score of 6 points with higher scores indicating more satisfaction with social support).

\subsubsection{Outcome variable}

The caregiving satisfaction was assessed using the Revised Caregiving Satisfaction Scale (RCSS) (Lawton, Moss, Hoffman, \& Perkinson, 2000). This scale is composed of 6 items, evaluating different aspects of PAC: (1) global satisfaction helping the relative; (2) feeling closer to the patient; (3) enjoying being with the patient; (4) boosting the caregiver's self-esteem; (5) delighting in the patient's pleasure; and (6) giving meaning to the caregiver's life. The RCSS is the most frequently used scale for measuring the positive aspects of caring (López et al., 2005).

\subsection{Statistical analysis}

Descriptive statistics (means, standard deviations, and percentages) were used to describe the sample. The correlations between the dependent variable (caregiving satisfaction) and the rest of the variables were performed. Pearson's correlation coefficient $\left(r_{x y}\right)$ and point-biserial correlation $\left(r_{p b}\right)$ were used to determine quantitative and dichotomous variables, respectively. The qualitative variables with more than two likely categories were dichotomized or converted to dummy variables. Only those variables that showed significant correlations with RCSS were introduced in a stepwise, multiple linear regression analysis to determine the best predictors of caregiving satisfaction. Following the SPM, only those variables significantly correlated were divided into three blocks and included the following in the regression analysis: first, the caregiver's background and context variables (caregiver age, type of relationship with the care recipient); second, the stress-related factors (care recipient cognitive impairment, burden, change in work shift, and need to leave work to take care of the relative); and third, caregiver mediators (support from the general practitioner and support from the dementia supervisor). To avoid multicollinearity problems (correlation between one of the independent variables and some linear combination of the remaining ones), condition indexes were considered, including only variables with indexes $\leq 15$. Moreover, the mean and standard deviation of the residual values were examined to ensure the accuracy of the model (mean $=0 ; \mathrm{SD}=1$ ). Furthermore, the Durbin and Watson (1951) test was applied to examine the independence of the residuals (value 2 for completely independent). However, comparisons among countries were not possible because of the limited sample size.

The software package IBM SPSS Statistics v.21.0 was used, and statistical significance was set at $p<0.05$.

\section{Results}

The descriptive characteristics of 101 caregivers of patients with dementia are included in Table 1. The age range of caregivers was 25-88 years; the majority of caregivers were female with a medium level of education $(46.5 \%)$ and married or living with someone $(82.2 \%)$. Regarding the type of relationship between the primary caregiver and the person with dementia, we found a similar proportion of caregivers with no consanguinity $(47.8 \%)$ and with consanguinity $(52.2 \%)$ relationship with the care recipient. There was also the same amount of employed and unemployed caregivers (50\% in each group). Additionally, the mean duration of caregiving was 64.26 months. 
Table 1. Characteristics of caregivers (caregiver's background and context, stress-related factors, mediators, and outcome)

Total

Caregiver's background and context

Age (mean, SD)

$61.28(12.61)$

Gender $(n, \%)$

Female

Male

30 (29.7)

Level of education $(n, \%)$

Low

21 (20.8)

Medium

47 (46.5)

High

Marital status $(n, \%)$

Without partner

With partner

83 (82.2)

Type of relationship $(n, \%)$

No consanguinity

44 (47.8)

Consanguinity

48 (52.2)

Employment status $(n, \%)$

Unemployed

$48(50.0)$

Employed

$48(50.0)$

Duration of caregiving (months as caregiver) (mean, SD)

64.26 (45.16)

Stress-related factors

Care recipient dementia severity $(n, \%)$

Moderate/moderately severe (GDS $4 / 5^{\mathrm{a}}$ )

Severe/very severe (GDS 6/7 ${ }^{\text {b }}$ )

44 (43.6)

57 (56.4)

Frequency of care (weekly hours) $(n, \%)$

$<20 \mathrm{~h}$

18 (17.8)

$\geq 20 \mathrm{~h}$

83 (82.2)

Subjective burden $-\mathrm{ZBI}^{\mathrm{c}}$ (mean, SD)

45.27 (12.17)

Likelihood of changing work shift $(n, \%)$

No

$82(82.8)$

Yes

17 (17.2)

Leave work to take care of the relative $(n, \%)$

No

78 (77.2)

Yes

$23(22.8)$

Caregiving competence $-\mathrm{CCS}^{\mathrm{d}}$ (mean, SD)

13.67 (1.86)

Mediators

Support from the general practitioner $(n, \%)$

No

62 (61.4)

Yes

39 (38.6)

Support from the dementia supervisor $(n, \%)$

No

65 (64.4)

Yes

36 (35.6)

Support from the relatives and friends $(n, \%)$

No

37 (36.6)

Yes

64 (63.4)

Support from the home services $(n, \%)$

No

$69(68.3)$

Yes

$32(31.7)$

Support from the respite care services $(n, \%)$

No

$92(91.1)$

Yes

$9(8.9)$

Support from the self-help groups $(n, \%)$ 
Table 1. Characteristics of caregivers (caregiver's background and context, stress-related factors, mediators, and outcome)

\begin{tabular}{ll}
\hline & Total \\
\hline No & $85(84.2)$ \\
Yes & $16(15.8)$ \\
Perceived social support ${ }^{\mathrm{e}}-\mathrm{SSQRS}$ (mean, SD) & $2.02(1.05)$ \\
Satisfaction with social support & $\mathrm{f}-\mathrm{SSQRS}(\mathrm{mean}, \mathrm{SD})$ \\
Outcome & $4.64(1.38)$ \\
Caregiving satisfaction - RCSS $^{\mathrm{g}}$ (mean, SD) & $20.72(6.04)$ \\
\hline
\end{tabular}

GDS = Global Deterioration Scale.

${ }^{a}$ GDS 4/5 indicates mild/moderate dementia.

${ }^{\mathrm{b}}$ GDS 6/7 indicates moderately-severe/severe dementia

${ }^{\mathrm{c}} \mathrm{ZBI}=$ Zarit Burden Interview. Maximum score of 88 points, with higher scores indicating an increased level of caregiver burden.

${ }^{\mathrm{d}} \mathrm{CCS}=$ Caregiving Competence Scale. Maximum score of 16 points, with higher scores indicating more feelings of competence. SSQRS = Social Support Questionnaire (Short-form).

${ }^{\mathrm{e}}$ Perceived social support sub-scale: maximum score of 9 points, with higher scores indicating more perceived social support.

${ }^{\mathrm{f}}$ Satisfaction with social support sub-scale: maximum score of 6 points, with higher scores indicating more satisfaction with social support.

${ }^{\mathrm{g}}$ RCSS = Revised Caregiving Satisfaction Scale. Maximum score of 30 points, with higher scores indicating greater satisfaction.

Regarding the stress-related factors, Table 1 shows that a substantial number of care recipients suffered from severe or very severe cognitive impairment (56.4\%). Approximately 82\% of caregivers reported spending more than $20 \mathrm{~h}$ per week in caring activities. The mean score for the ZBI was 45.27 with $17.2 \%$ of the sample having to change the work shift to look after their elder and $22.8 \%$ of the caregivers leaving work to take care of the relative. The caregiving competence mean score was 13.67.

Considering mediators, the support provided by the general practitioner was used by $38.6 \%$ of the caregivers; support from the dementia supervisor was used by $35.6 \%$ of the caregivers; support from relatives and friends was identified by $63.4 \%$ of the caregivers; support from home services was used by $31.7 \%$ of the caregivers; support from respite care services was used by $8.9 \%$ of the caregivers; and support from self-help groups was used by $15.8 \%$ of the caregivers. The mean values were 2.02 for perceived social support and 4.64 for satisfaction with that support.

The mean value for the outcome of caregiving satisfaction, as measured using RCSS, was 20.72.

The correlations were calculated between the caregiving satisfaction outcome and the caregiver's background and context variables, stress-related factors, and caregiver's mediators. Table 2 shows that eight of the twenty-two studied variables showed a significant correlation with caregiving satisfaction, as follows: caregiver age, type of relationship, care recipient dementia severity, subjective burden, change in work shift, leaving work to take care of the relative, support from the general practitioner, and support from the dementia supervisor. 
Table 2. Correlations between caregiving satisfaction and caregiver's background and context variables, stress-related factors, and mediators.

Correlation coefficients

$\left(r_{x y}\right.$ or $\left.r_{p b}\right)$

Caregiver's background and context

1. Age

$-0.238^{*}$

2. Gender $(0=$ female; $1=$ male $)$

$-0.010$

3. Low level of education $(0=$ no; $1=$ yes $)$

0.056

4. Medium level of education $(0=$ no; $1=$ yes $)$

$-0.069$

5. High level of education $(0=$ no; $1=$ yes $)$

0.025

6. Marital status $(0=$ without partner; $1=$ with partner)

$-0.142$

7. Type of relationship $(0=$ no consanguinity; $1=$ consanguinity $) \quad 0.269^{* *}$

8. Employment status $(0=$ unemployed; $1=$ employed $) \quad 0.121$

9. Duration of caregiving (months as caregiver) $\quad-0.039$

Stress-related factors

10. Care recipient dementia severity $(0=$ GDS $4 / 5 ; 1=$ GDS $6 / 7) \quad 0.285^{* *}$

11. Frequency of care (weekly hours) $(0=\langle 20 ; 1=\geq 20) \quad 0.159$

12. Subjective burden (ZBI) $\quad-0.211^{*}$

13. Likelihood of changing work shift $(0=$ no; $1=$ yes $) \quad 0.249^{*}$

14. Leave work to take care of the relative $(0=$ no; $1=$ yes $) \quad 0.222^{*}$

15. Caregiving competence (CCS) 0.147

Caregivers mediators

16. Support from the general practitioner $(0=$ no; $1=$ yes $) \quad 0.243^{*}$

17. Support from the dementia supervisor $(0=$ no; $1=$ yes $) \quad-0.210^{*}$

18. Support from the relatives and friends $(0=$ no; $1=$ yes $) \quad 0.020$

19. Support from the home services $(0=$ no; $1=$ yes $) \quad-0.124$

20. Support from the respite care services $(0=$ no; $1=$ yes $) \quad-0.043$

21. Support from the self-help groups $(0=$ no; $1=$ yes $) \quad-0.039$

22. Perceived social support (SSQRS) 0.103

23. Satisfaction with social support (SSQRS) $\quad 0.166$

GDS = Global Deterioration Scale; ZBI = Zarit Burden Interview; CCS = Caregiving Competence Scale; SSQRS = Social Support Questionnaire (Short-form).

$* p<0.05$.

$* * p<0.01$

Variables that significantly correlated with caregiving satisfaction were entered in a stepwise, multiple linear regression analysis (Table 3). The Durbin-Watson test value (1.700) was nearly 2. The regression analysis showed that higher caregiving satisfaction was significantly predicted by having a consanguinity relationship with the care recipient, with suffering lower levels of subjective burden, and with the care recipient having severe or very severe dementia. The coefficient of determination $\left(R^{2}\right)$ and the significant $F$ test $(p<0.001)$ of the combined effects of these three predictors simultaneously shown in the third model would explain $20 \%$ of the caregiving satisfaction (adjusted $R^{2}$ ). No harmful multicollinearity was detected. 
Table 3. Stepwise, multiple linear regression analysis for variables predicting caregiving satisfaction $(n=88)$.

\begin{tabular}{|c|c|c|c|c|}
\hline & Model 1 & Model 2 & \multicolumn{2}{|c|}{ Model 3} \\
\hline & $\beta$ & $\beta$ & $\beta$ & $t$ \\
\hline \multicolumn{5}{|l|}{ Independent variables } \\
\hline $\begin{array}{l}\text { Type of relationship }(0=\text { no consanguinity; } \\
1=\text { consanguinity) }\end{array}$ & $2.59^{* *}$ & $3.15^{* * *}$ & 0.28 & $2.88^{* *}$ \\
\hline Subjective burden (ZBI) & & $-3.25^{* * *}$ & -0.31 & $-3.25^{* * *}$ \\
\hline $\begin{array}{l}\text { Care recipient dementia severity }(0=\text { GDS } \\
4 / 5 ; 1=\text { GDS } 6 / 7)\end{array}$ & & & 0.23 & $2.44^{*}$ \\
\hline Total $R^{2}$ & 0.07 & 0.17 & & \\
\hline Adjusted $R^{2}$ & 0.06 & 0.06 & & \\
\hline$R^{2}$ change & 0.07 & 0.07 & & \\
\hline$F$ for $R^{2}$ change & $F_{1,86}=6.70^{* *}$ & $F_{1,85}=10.57^{* *}$ & & $.98^{* *}$ \\
\hline Total $F$ & $F_{1,86}=6.70^{* *}$ & $F_{2,85}=9.01^{* * *}$ & $F_{3,}$ & $.35^{* * *}$ \\
\hline
\end{tabular}

$\mathrm{ZBI}=$ Zarit Burden Interview; GDS = Global Deterioration Scale

$* p<0.05$.

$* * p<0.01$.

$* * * p<0.001$.

\section{Discussion}

Numerous authors have attempted to identify which factors influence the caregiver' negative outcomes, including characteristics of the caregiver and the care recipient, situational aspects, and other factors that may act as buffers or mediate in this process (Bertrand et al., 2006, Gaugler et al., 2009, González-Abraldes et al., 2013, Losada et al., 2010 and Smith et al., 2011). However, little is known about the factors that mediate or promote positive outcomes of caregiving, as the satisfaction with caregiving. Given the proven influence of PAC on the well-being of the caregivers (Cohen et al., 1994, Cohen et al., 2002, Lawton et al., 1991, Mausbach et al., 2004, Motenko, 1989 and Noonan and Tennstedt, 1997) the current study expands the knowledge of the likely predictors of caregiving satisfaction by assessing which factors influence this positive aspect of caring, using the stress process model of Pearlin's et al. (1990) as a conceptual frame in which to explain the results.

According to the results of the regression model, we found that the predictors of the caregiver's satisfaction spanned two of the three domains outlined by the SPM, one feature of caregiver's background and two variables of stress-related factors. To be more specific, the analysis showed that having a consanguinity relationship with the care recipient, suffering from lower levels of subjective burden, and the care recipient having severe or very severe dementia are the most important predictors of higher caregiving satisfaction.

Previous research found that having a consanguinity relationship between the caregiver and the care recipient positively influences the health of the caregiver (Marks, Lambert, \& Choi, 2002) and reduces depressive symptomatology (Noonan \& Tennstedt, 1997) and stress (Compton, Flanagan, \& Greg, 1997). Consistent with these previous results, our findings posit that having a consanguinity relationship not only influences the health of the caregiver but is also an important factor when predicting caregiving satisfaction.

Similar to other researchers (López et al., 2005 and Neubauer et al., 2008), we found that caregiving for a relative with dementia is a time-consuming activity, with the majority of the caregivers giving assistance more than $20 \mathrm{~h}$ per week during a mean time period of about five years. However, in agreement with others (López et al., 2005), we found that neither the duration of caregiving (the months as caregiver) nor the frequency of care (weekly hours as a caregiver), significantly correlated with satisfaction in this study. The lack of relation between satisfaction and the duration of caregiving is not surprising, since this result has been found in several studies (Cohen et al., 1994, López et al., 2005 and Picot, 1995). Nevertheless, among the stress-related factors, the relation between the frequency of care and satisfaction is a common result in this field, suggesting greater satisfaction as more time consumed in caregiving activities (Kinney et al., 1995, Lawton et al., 1991 and Quinn et al., 2012). 
Although this relation was not found in the present study, the care recipient dementia severity was a predictor of satisfaction. Specifically, having a more severe GDS level (stages 6 and 7) correlated positively with higher satisfaction, in accordance with Andrén and Elmståhl (2005) who found that satisfaction, expressed as purpose, was influenced by the patient's severity of disease, concluding that this may reflect an increasing understanding of the disease process.

These results are in contrast with previous research showing that as the illness progresses, the satisfaction with the caregiver role decreases (Holst \& Edberg, 2011), thus influencing the intensity of the caregiver's grief (Warchol-Biedermann et al., 2014).

Related to the frequency of care, some studies have found more positive appraisals or greater satisfactions in the caregiving experience associated with greater functional impairments among care recipients (Kramer, 1993, Lawton et al., 1989 and Lawton et al., 1991). Patients with dementia in a mild or moderate stage of GDS level usually have better functioning state than patients with severe/very severe dementia, and this may allow the patient to use a rich variety of strategies in everyday life and cope with the hardships (Keady \& Nolan, 1994).

Consequently, in mild to moderate stages of dementia the caregiver might not be as implicated in the caregiving process as in later stages, thus maintaining a close relationship with his or her relative, but not as intense. As the illness progresses, the relationship between the caregiver and the patient might become more intense, and the caregiver might assume a more involved position, reaching expectancies of control, particularly self-efficacy and contingency (Contador, Fernández-Calvo, Palenzuela, Miguéis, \& Ramos, 2012). This new or renewed relationship with the loved one, together with these new expectancies, could be the reason why the caregiving satisfaction increases as GDS level progresses. However, it would be fruitful to investigate in future studies the contribution of the different variables included in Pearlin's model to the six different domains of the construct satisfaction included in the RCSS, i.e. the sense of satisfaction from helping the care recipient, feeling of closeness, enjoyment, increase in self-esteem, pleasure and reciprocity, and meaning in life. This would allow achieving a better understanding of our results, given that the predictor variables used in the current study might influence these domains in different ways.

Another aspect that might improve the feeling of satisfaction as the GDS level increases could be the feeling of "growing as a person" that the caregivers might feel and the development of new skills and competence as caregivers (Sequeira, 2013). In fact, higher sense of caregiving competence has been found in several studies as a predictive factor of PAC or satisfaction in the caregiver role (Carbonneau et al., 2010 and Quinn et al., 2012). Although no correlation was found between the sense of competence and satisfaction in the present study, the mean score in competence was very high.

This high sense of competence and the greater satisfaction in more severe levels of dementia observed in our caregivers, might also be related with the reduction of the behavioral and psychological symptoms in dementia (BPSD), since as was previously addressed, the BPSD eventually decreases at advanced stages of the disease (Cummings, 2003). In sum, it seems that more help with basic daily care activities may be more intimate and therefore more rewarding in comparison with responding to behavioral disturbances (Hilgeman et al., 2007).

Our data indicated that suffering a subjective burden is an important factor predicting negative characteristics of the outcome of caregiving satisfaction. Caregivers who perceive their role as a burden, experience lower levels of satisfaction. This concept is consistent with the literature dating to the 1980s (George and Gwyther, 1986 and Zarit et al., 1980), which claimed that caring for persons diagnosed with dementia in the community placed a major burden on caregivers, resulting in adverse psychological and physical consequences in caregivers. Additionally, Cohen et al. (2002) showed that the presence of few positive aspects is an indicator that caregivers will be at risk for being overwhelmed by their caring situation and therefore will be more likely to report depression, burden or poor health.

Again, this result might be explained by the decrease in the BPSD as the dementia severity progresses. BPSD are the primary determinants of burden in dementia caregivers (Allegri et al., 2006, Cheng et al., 2012, Gaugler et al., 2000 and Pinquart and Sörensen, 2003). In a previous study, Cheng, Lam, Kwok, $\mathrm{Ng}$, and Fung (2013) found at the same time a positive association between BPSD and burden and a negative and independent association between BPSD and positive gains. Then, it is possible that the decrease of BPSD in more severe stages of dementia leads to a decrease in burden and to an increase in the level of satisfaction as a caregiver.

The model of Lawton et al. (1991) demonstrated that among adult children, high levels of caregiving behavior (frequency of assistance in personal-care tasks) can result in both greater caregiving satisfaction and burden. This profile is quite similar to the general profile of the caregiver included in the current study: mainly females, adult children of the care recipient, with medium level of education, with high levels of caregiving behavior since they mainly care for a care recipient in severe stages of the disease, with a moderate level of subjective burden, high sense of competence, and high levels of satisfaction. 
Taking all these data together it is possible that our sample was made up by a very select group of caregivers. In an interesting study, Robertson et al. (2007) found an atypical group of caregivers, named the "intense affect group" since they presented at the same time high negative and positive affect. They found that this group was characterized by being mainly females, experiencing higher caregiving rewards and also a relatively elevated level of subjective stress, as was our case. Robertson et al. (2007) concluded that caregivers in the intense group may be psychologically resilient individuals who are able to cultivate and summon positive emotions in the wake of stressful experiences. Therefore, it seems necessary to establish different subsets of caregivers, especially those more atypical and analyze the factors associated with caregiving satisfaction in each of them in order to make more adapted interventions. The stress process model (Pearlin et al., 1990) posits that social support is an important element acting as a mediator among the stressors that significantly influence and address individual differences in the caregiver outcomes. Contrary to what we have anticipated, none of the mediators investigated in the current study played a role in predicting caregiving satisfaction. This surprising result could be explained because it is not the actual social support or network size that predicts the caregiver's well-being but the perceived support. Among the social resources that the caregivers are queried about, we recognize that several of social resources could be considered as actual social support, whereas others are better interpreted as perceived social support. We assume that this type of discordance may have diminished these unexpected results because it is known that caregivers' subjective well-being and mental health improve when they receive support (Millán-Calenti et al., 2000).

The strength of this exploratory study is that it focuses on a little explored area of caregiving, that is, the potential source of personal satisfaction experienced by many caregivers. Although the study provides important findings, likely limitations include its cross-sectional design, the nonrandom selection of subjects, the small sample size and the inclusion of caregivers from three different countries, thus making it difficult to analyze country differences in the multivariate phase of the analysis. However, we followed rigid criteria and maximum control when selecting the sample in the places where we conducted the study.

In addition, and given the weak variance explained by our regression model, it is possible that other predictors not studied here but frequently addressed in the literature about caregiving satisfaction, might be stronger predictors, such as the caregiver health status (Andrén and Elmståhl, 2005 and Kramer, 1993), the quality of prior relationship (Kramer, 1993, López et al., 2005 and Motenko, 1989), the use of coping strategies (López et al., 2005 and Sequeira, 2013), and other caregiver characteristics such as personality traits (Koerner, Kenyon, \& Shirai, 2009).

Future investigations will hopefully include larger and probabilistic samples in addition to these missing variables, which will permit an in-depth exploration of the linear regression model and will ensure greater statistical power.

\section{Conclusion}

In accordance with previous literature, caregivers are more likely to experience negative consequences if they feel less satisfaction from their role of caregiver. Therefore, it seems important to identify predictors of satisfaction with caregiving that permit to design psychosocial interventions focused on enhancement of the positive aspects of caring. Taking into account the predictors of caregiving satisfaction found in the present study, these interventions should be especially aimed at caregivers without a consanguinity relationship with the care recipients, with high levels of subjective burden, and at those managing care recipients with mild or moderate stages of dementia. From the current data, we consider that a possible effective intervention in these caregivers might be their inclusion in psychoeducational programs, aimed to increase the understanding of the disease, especially of the behavioral and psychological symptoms of dementia.

\section{Conflict of interest statement}

None.

\section{Acknowledgments}

We thank the day care centers, associations and caregivers from Spain, Denmark, and Poland for participating in the study and helping to increase the knowledge of caregiving satisfaction.

This work was supported by the research project "understAID: a platform that helps informal caregivers to understand and aid their demented relatives" (grant number AAL-2012-5-107), founded by the European Commission in the Ambient Assisted Living (AAL) Joint Programme and national funding 
agencies [Ministry of Industry, Energy and Tourism/Ministerio de Industria, Energía y Turismo (Spain); National Institute of Health Carlos III/Instituto de Salud Carlos III (Spain); Danish Agency for Science, Technology and Innovation (Denmark); and Centre for Research and Development/Narodowe Centrum Badań i Rozwoju (Poland)].

\section{References}

Allegri et al., 2006. R.F. Allegri, D. Sarasola, C.M. Serrano, F.E. Taragano, R.L. Arizaga, J. Butman, et al. Neuropsychiatric symptoms as a predictor of caregiver burden in Alzheimer's disease. Neuropsychiatric Diseases and Treatment, 2 (2006), pp. 105-110.

M.E. Alspaugh, M.A. Stephens, A.L. Townsend, S.H. Zarit, R. Greene. Longitudinal patterns of risk for depression in dementia caregivers: Objective and subjective primary stress as predictors. Psychology and Aging, 14 (1999), pp. 34-43 http://dx.doi.org/10.1037//0882-7974.14.1.34.

American Psychiatric Association, 2000. American Psychiatric Association. Diagnostic and statistical manual of mental disorders (4th ed., text revision). American Psychiatric Association, Washington, DC (2000).

Andrén and Elmståhl, 2005. S. Andrén, S. Elmståhl. Family caregivers' subjective experiences of satisfaction in dementia care: Aspects of burden, subjective health and sense of coherence. Scandinavian Journal of Caring Science, 19 (2005), pp. 157-168 http://dx.doi.org/10.1111/j.1471-6712.2005.00328.x.

Anthony-Bergstone et al., 1988. C.R. Anthony-Bergstone, S.H. Zarit, M. Gatz. Symptoms of psychological distress among caregivers of dementia patients. Psychology and Aging, 3 (1988), pp. 245-248 http://dx.doi.org/10.1037/0882-7974.3.3.245.

Bertrand et al., 2006. R.M. Bertrand, L. Fredman, J. Saczynski. Are all caregivers created equal? Stress in caregivers to adults with and without dementia. Journal of Aging and Health, 18 (2006), pp. 534-551 http://dx.doi.org/10.1177/0898264306289620.

Brodaty and Donkin, 2009. H. Brodaty, M. Donkin. Family caregivers of people with dementia. Dialogues in Clinical Neurosciences, 11 (2009), pp. 217-228.

Carbonneau et al., 2010. H. Carbonneau, C. Caron, J. Desrosiers. Development of a conceptual framework of positive aspects of caregiving in dementia. Dementia, 9 (2010), pp. 327-353 http://dx.doi.org/10.1177/1471301210375316.

Cheng et al., 2012. S.T. Cheng, T. Kwok, L.C. Lam. Neuropsychiatric symptom clusters of Alzheimer's disease in Hong Kong Chinese: Prevalence and confirmatory factor analysis of the Neuropsychiatric Inventory. International Psychogeriatrics, 24 (2012), pp. 1465-1473 http://dx.doi.org/10.1017/S1041610212000609.

Cheng et al., 2013. S.T. Cheng, L.C. Lam, T. Kwok, N.S. Ng, A.W. Fung. The social networks of Hong Kong Chinese family caregivers of Alzheimer's disease: Correlates with positive gains and burden. The Gerontologist, 53 (2013), pp. 998-1008 http://dx.doi.org/10.1093/geront/gns195

Cohen et al., 2002. C.A. Cohen, A. Colantonio, L. Vernich. Positive aspects of caregiving: Rounding out the caregiver experience. International Journal of Geriatric Psychiatry, 17 (2002), pp. 184-188 http://dx.doi.org/10.1002/gps.561.

Cohen et al., 1994. C.A. Cohen, D. Pushkar Gold, K.I. Shulman, C.A. Zucchero. Positive aspects in caregiving: An overlooked variable in research. Canadian Journal on Aging, 13 (1994), pp. 378-391 http://dx.doi.org/10.1017/S071498080000619X.

Compton et al., 1997. S.A. Compton, P. Flanagan, W. Gregg. Elder abuse in people with dementia in Northern Ireland: Prevalent and predictor in cases referred to psychiatry of old age service. International Journal of Geriatric Psychiatry, 12 (1997), pp. 632-635 http://dx.doi.org/10.1002/(SICI)1099-1166(199706)12:6<632::AIDGPS570>3.0.CO;2-9.

Contador et al., 2012. I. Contador, B. Fernández-Calvo, D.L. Palenzuela, S. Miguéis, F. Ramos. Prediction of burden in family caregivers of patients with dementia: A perspective of optimism based on generalized expectancies of control. Aging \& Mental Health, 16 (2012), pp. 675-682 http://dx.doi.org/10.1080/13607863.2012.684666

Cooper et al., 2007. C. Cooper, T.B. Balamurali, G. Livingston. A systematic review of the prevalence and covariates of anxiety in caregivers of people with dementia. International Psychogeriatrics, 19 (2007), pp. 175-195 http://dx.doi.org/10.1017/S1041610206004297.

Crespo and Fernández-Lansac, 2013. M. Crespo, V. Fernández-Lansac. Factors associated with anger and anger expression in caregivers of elderly relatives. Aging \& Mental Health, 18 (2013), pp. 454-462 http://dx.doi.org/10.1080/13607863.2013.856857.

Cummings, 2003. J.L. Cummings. The neuropsychiatry of Alzheimer's disease and related dementias. Martin Dunitz, London (2003).

Durbin and Watson, 1951. J. Durbin, G.S. Watson. Testing for serial correlation in least squares regression. II. Biometrika, 38 (1951), pp. 159-177 http://dx.doi.org/10.2307/2332325

Gaugler et al., 2000. J.E. Gaugler, A. Davey, L.I. Pearlin, S.H. Zarit. Modeling caregiver adaptation over time: The longitudinal impact of behavior problems. Psychology and Aging, 15 (2000), pp. 437-450 http://dx.doi.org/10.1037/0882-7974.15.3.437.

Gaugler et al., 2009. J.E. Gaugler, M.S. Mittelman, K. Hepburn, R. Newcomer. Predictors of change in caregiver burden and depressive symptoms following nursing home admission. Psychology and Aging, 24 (2009), pp. 385 $396 \mathrm{http} / / / \mathrm{dx}$. doi.org/10.1037/a0016052. 
George and Gwyther, 1986. L. George, L. Gwyther. Caregiver well-being: A multidimensional examination of family caregivers of demented adults. The Gerontologist, 26 (1986), pp. 253-259 http://dx.doi.org/10.1093/geront/26.3.253.

González-Abraldes et al., 2013. I. González-Abraldes, J.C. Millán-Calenti, L. Lorenzo-López, A. Maseda. The influence of neuroticism and extraversion on the perceived burden of dementia caregivers: An exploratory study. Archives of Gerontology and Geriatrics, 56 (2013), pp. 91-105 http://dx.doi.org/10.1016/j.archger.2012.07.011.

Haley et al., 1987. W.E. Haley, E.G. Levine, S.L. Brown, A.A. Bartolucci. Stress, appraisal, coping, and social support as predictors of adaptational outcome among dementia caregivers. Psychology and Aging, 2 (1987), pp. 323-330 http://dx.doi.org/10.1037/0882-7974.2.4.323.

Hilgeman et al., 2007. M.M. Hilgeman, R.S. Allen, J. DeCoster, L.D. Burgio. Positive aspects of caregiving as a moderator of treatment outcome over 12 months. Psychology and Aging, 22 (2007), pp. 361-371 http://dx.doi.org/10.1037/0882-7974.22.2.361.

Holst and Edberg, 2011. G. Holst, A.K. Edberg. Wellbeing among people with dementia and their next of kin over a period of 3 years. Scandinavian Journal of Caring Sciences, 25 (2011), pp. 549-557 http://dx.doi.org/10.1111/j.1471-6712.2010.00863.x.

Keady and Nolan, 1994. J. Keady, M. Nolan. Younger onset dementia: Developing a longitudinal model as the basis for a research agenda and as a guide to interventions with sufferers and carers. Journal of Advanced Nursing, 19 (1994), pp. 659-669 http://dx.doi.org/10.1111/j.1365-2648.1994.tb01136.x.

Kiecolt-Glaser et al., 1991. J.K. Kiecolt-Glaser, J.R. Dura, C.E. Speicher, O.J. Trask, R. Glaser. Spousal caregivers of dementia victims: Longitudinal changes in immunity and health. Psychosomatic Medicine, 53 (1991), pp. 345362.

Kinney et al., 1995. J.M. Kinney, M.A.P. Stephens, M.M. Franks, V.K. Norris. Stresses and satisfactions of family caregivers to older stroke patients. The Journal of Applied Gerontology, 74 (1995), pp. 3-21 http://dx.doi.org/10.1177/073346489501400101.

Koerner et al., 2009. S.S. Koerner, D.B. Kenyon, Y. Shirai. Caregiving for elder relatives: Which caregivers experience personal benefits/gains?. Archives of Gerontology and Geriatrics, 48 (2009), pp. 238-245 http://dx.doi.org/10.1016/j.archger.2008.01.015.

Kramer, 1993. B.J. Kramer. Marital history and the prior relationship as predictors of positive and negative outcomes among wife caregivers. Family Relations, 42 (1993), pp. 367-375 http://dx.doi.org/10.2307/585336

Kramer, 1997. B.J. Kramer. Gain in the caregiving experience: Where are we? What next?. The Gerontologist, 37 (1997), pp. 218-232 http://dx.doi.org/10.1093/geront/37.2.218.

Lawton et al., 1989. M.P. Lawton, M.H. Kleban, M. Moss, M. Rovine, A. Glicksman. Measuring caregiving appraisal. The Journals of Gerontology. Series B, Psychological Sciences and Social Sciences, 44 (1989), pp. P61-P71 http://dx.doi.org/10.1093/geronj/44.3.P61

Lawton et al., 2000. M.P. Lawton, M. Moss, C. Hoffman, M. Perkinson. Two transitions in daughter's caregiving careers. The Gerontologist, 40 (2000), pp. 437-448 http://dx.doi.org/10.1093/geront/40.4.437.

Lawton et al., 1991. M.P. Lawton, M. Moss, M.H. Kleban, A. Glicksman, M. Rovine. A two-factor model of caregiving appraisal and psychological well-being. The Journal of Gerontology, 46 (1991), pp. 181-189 http://dx.doi.org/10.1093/geronj/46.4.P181

López et al., 2005. J. López, J. López-Arrieta, M. Crespo. Factors associated with the positive impact of caring for elderly and dependent relatives. Archives of Gerontology and Geriatrics, 41 (2005), pp. 81-94 http://dx.doi.org/10.1016/j.archger.2004.12.001.

Losada et al., 2010. A. Losada, A. Pérez-Peñaranda, E. Rodríguez-Sánchez, M.A. Gómez-Marcos, C. BallesterosRíos, I.R. Ramos-Carrera, et al. Leisure and distress in caregivers for elderly patients. Archives of Gerontology and Geriatrics, 50 (2010), pp. 347-350 http://dx.doi.org/10.1016/j.archger.2009.06.001.

Lundh, 1999. U. Lundh. Family carers. 2: Sources of satisfaction among Swedish carers. British Journal of Nursing, 8 (1999), pp. 647-652 http://dx.doi.org/10.12968/bjon.1999.8.10.6601.

Marks et al., 2002. N.F. Marks, J.D. Lambert, H. Choi. Transitions to caregiving, gender, and psychological wellbeing: A prospective U.S. national study. Journal of Marriage and Family, 64 (2002), pp. 657-667 http://dx.doi.org/10.1111/j.1741-3737.2002.00657.x

Mausbach et al., 2004. B.T. Mausbach, D.W. Coon, C. Depp, Y.G. Rabinowitz, E. Wilson-Arias, H.C. Kraemer, et al. Ethnicity and time to institutionalization of dementia patients: A comparison of Latina and Caucasian female family caregivers. Journal of the American Geriatrics Society, 52 (2004), pp. 1077-1084 http://dx.doi.org/10.1111/j.1532-5415.2004.52306.x.

Mausbach et al., 2010. B.T. Mausbach, S.K. Roepke, M.G. Ziegler, M. Milic, R. von Känel, J.E. Dimsdale, et al. Association between chronic caregiving stress and impaired endothelial function in the elderly. Journal of the American College of Cardiology, 55 (2010), pp. 2599-2606 http://dx.doi.org/10.1016/jjacc.2009.11.093.

McKhann et al., 1984. G. McKhann, D. Drachman, M. Folstein, R. Katzman, D. Price, E.M. Stadlan. Clinical diagnosis of Alzheimer's disease: Report of the NINCDS-ADRDA Work Group under the auspices of Department of Health and Human Services Task Force on Alzheimer's Disease. Neurology, 34 (1984), pp. 939944 http://dx.doi.org/10.1212/WNL.34.7.939.

Millán-Calenti et al., 2000. J.C. Millán-Calenti, M. Gandoy-Crego, M. Antelo-Martelo, M. López-Martinez, M.P. Riveiro-López, J.M. Mayán-Santos. Helping the family carers of Alzheimer's patients: From theory...to practice. A preliminary study. Archives of Gerontology and Geriatrics, 30 (2000), pp. 131-138 http://dx.doi.org/10.1016/S0167-4943(00)00044-3.

Motenko, 1989. A.K. Motenko. The frustrations, gratification, and well-being of dementia caregivers. The Gerontologist, 29 (1989), pp. 166-172 http://dx.doi.org/10.1093/geront/29.2.166. 
Neubauer et al., 2008. S. Neubauer, R. Holle, P. Menn, M. Grossfeld-Schmitz, E. Graesel. Measurement of informal care time in a study of patients with dementia. International Psychogeriatrics, 20 (2008), pp. 1160-1176 http://dx.doi.org/10.1017/S1041610208007564

Noonan and Tennstedt, 1997. A.E. Noonan, S.T. Tennstedt. Meaning in caregiving and its contribution to caregiver well-being. The Gerontologist, 37 (1997), pp. 785-794 http://dx.doi.org/10.1093/geront/37.6.785.

Pearlin et al., 1990. L.I. Pearlin, J.T. Mullan, S.J. Semple, M.M. Skaff. Caregiving and the stress process: An overview of concepts and their measures. The Gerontologist, 30 (1990), pp. 583-594 http://dx.doi.org/10.1093/geront/30.5.583.

Pinquart and Sörensen, 2003. M. Pinquart, S. Sörensen. Associations of stressors and uplifts of caregiving with caregiver burden and depressive mood: A meta-analysis. Journal of Gerontology: Psychological Sciences, 58 (2003), pp. 112-128 http://dx.doi.org/10.1093/geronb/58.2.P112.

Picot, 1995. S.J. Picot. Choice and social exchange theory and the rewards of African American caregivers. Journal of National Black Nurses Association, 7 (1995), pp. 29-40.

Pruchno et al., 1990. R.A. Pruchno, J.E. Michaels, S.L. Potashnik. Predictors of institutionalization among Alzheimer disease victims with caregiving spouses. The Journal of Gerontology, 45 (1990), pp. S259-S266 http://dx.doi.org/10.1093/geronj/45.6.S259.

Quinn et al., 2012. C. Quinn, L. Clare, R.T. Woods. What predicts whether caregivers of people with dementia find meaning in their role?. International Journal of Geriatric Psychiatry, 27 (2012), pp. 1195-1202 http://dx.doi.org/10.1002/gps.3773.

Reisberg et al., 1982. B. Reisberg, S.H. Ferris, M.D. De Leon, T. Crook. The global deterioration scale for assessment of primary degenerative dementia. The American Journal of Psychiatry, 139 (1982), pp. 1136-1139.

Robertson et al., 2007. S.M. Robertson, S.H. Zarit, L.G. Duncan, M.J. Rovine, E.E. Femia. Family caregivers' patterns of positive and negative affect. Family Relations, 56 (2007), pp. 12-23 http://dx.doi.org/10.1111/j.17413729.2007.00436.x.

Roepke et al., 2011. S.K. Roepke, B.T. Mausbach, T.L. Patterson, R. von Känel, S. Ancoli-Israel, A.L. Harmell, et al. Effects of Alzheimer caregiving on allostatic load. Journal of Health Psychology, 16 (2011), pp. 58-69 http://dx.doi.org/10.1177/1359105310369188

Sarason et al., 1987. I. Sarason, B. Sarason, E. Shearin, G. Pierce. A brief measure of social support: Practical and theoretical implications. Journal of Social and Personal Relationships, 4 (1987), pp. 497-510 http://dx.doi.org/10.1177/0265407587044007.

Schreiner et al., 2006. A.S. Schreiner, T. Morimoto, Y. Arai, S. Zarit. Assessing family caregiver's mental health using a statistically derived cut-off score for the Zarit Burden Interview. Aging \& Mental Health, 10 (2006), pp. 107-111 http://dx.doi.org/10.1080/13607860500312142.

Schulz et al., 2005. R. Schulz, L.M. Martire, J.N. Klinger. Evidence-based caregiver interventions in geriatric psychiatry. Psychiatric Clinics of North America, 28 (2005), pp. 1007-1038 http://dx.doi.org/10.1016/j.psc.2005.09.003.

Schulz et al., 1997. R. Schulz, J.T. Newsom, K. Fleissner, A.R. de Camp, A.P. Nieboer. The effects of bereavement after family caregiving. Aging \& Mental Health, 1 (1997), pp. 269-282 http://dx.doi.org/10.1080/13607869757173.

Sequeira, 2013. C. Sequeira. Difficulties, coping strategies, satisfaction and burden in informal Portuguese caregivers. Journal of Clinical Nursing, 22 (3-4) (2013), pp. 491-500 http://dx.doi.org/10.1111/jocn.12108.

Smith et al., 2011. G.R. Smith, G.M. Williamson, L.S. Miller, R. Schulz. Depression and quality of informal care: A longitudinal investigation of caregiving stressors. Psychology and Aging, 26 (2011), pp. 584-591 http://dx.doi.org/10.1037/a0022263.

Tarlow et al., 2004. B.J. Tarlow, S.R. Wisniewski, S.H. Belle, M. Rubert, M.G. Ory, D. Gallaguer-Tomson. Positive aspects of caregiving: Contributions of the REACH project to the development of new measures for Alzheimer's caregiving. Research on Aging, 26 (2004), pp. 429-453 http://dx.doi.org/10.1177/0164027504264493.

Vitaliano et al., 2011. P.P. Vitaliano, M. Murphy, H.M. Young, D. Echeverria, S. Borson. Does caring for a spouse with dementia promote cognitive decline? A hypothesis and proposed mechanisms. Journal of American Geriatric Society, 59 (2011), pp. 900-908 http://dx.doi.org/10.1111/j.1532-5415.2011.03368.x.

Vitaliano et al., 2003. P.P. Vitaliano, J. Zhang, J.M. Scanlan. Is caregiving hazardous to one's physical health? A meta-analysis. Psychological Bulletin, 129 (2003), pp. 946-972 http://dx.doi.org/10.1037/0033-2909.129.6.946.

von Känel et al., 2012. R. von Känel, P.J. Mills, B.T. Mausbach, J.E. Dimsdale, T.L. Patterson, M.G. Ziegler, et al. Effect of Alzheimer caregiving on circulating levels of C-reactive protein and other biomarkers relevant to cardiovascular disease risk: A longitudinal study. Gerontology, 58 (2012), pp. 354-365 http://dx.doi.org/10.1159/000334219.

Warchol-Biedermann et al., 2014. K. Warchol-Biedermann, E. Mojs, R. Gregersen, K. Maibom, J.C. Millán-Calenti, A. Maseda. What causes grief in dementia caregivers?. Archives of Gerontology and Geriatrics, 59 (2014), pp. 462-467 http://dx.doi.org/10.1016/j.archger.2014.05.013.

Wilson, 1989. H.S. Wilson. Family caregiving for a relative with Alzheimer's dementia: Coping with negative choices. Nursing Research, 38 (2) (1989), pp. 94-98 http://dx.doi.org/10.1097/00006199-198903000-00011.

World Health Organization, 1992. World Health Organization. ICD-10 classifications of mental and behavioral disorders: Clinical descriptions and diagnostic guidelines. (1992) Retrieved from http://www.who.int/classifications/icd/en/bluebook.pdf.

World Health Organization and Alzheimer's Disease International, 2012. World Health Organization, Alzheimer's Disease International. Mental health publications. Dementia: A public health priority. (2012) Retrieved from http://www.who.int/mental_health/publications/dementia_report_2012/en. 
Zarit et al., 1985. S.H. Zarit, N.K. Orr, J.M. Zarit. The hidden victims of Alzheimer's disease: Families under stress. New York University Press, New York (1985).

Zarit et al., 1980. S.H. Zarit, K.E. Reever, J. Bach-Peterson. Relatives of the impaired elderly: Correlates of feelings of burden. The Gerontologist, 20 (1980), pp. 649-655 http://dx.doi.org/10.1093/geront/20.6.649. 